\title{
Effect of Rotational Speed Modulation on the Weakly Nonlinear Heat Transfer in Walter-B Viscoelastic Fluid in the Highly Permeable Porous Medium
}

\author{
Anand Kumar ${ }^{1}$, Vinod K. Gupta ${ }^{2}$, Neetu Meena ${ }^{1}$ and Ishak Hashim ${ }^{3, *(\mathbb{D})}$ \\ 1 Department of Mathematics, Central University of Rajasthan, Ajmer 305817, India; \\ aanandbhu@gmail.com (A.K.); nitu95meena@gmail.com (N.M.) \\ 2 Department of Mathematics, DIT University, Dehradun 248009, India; vinodguptabhu@gmail.com \\ 3 Department of Mathematical Sciences, Faculty of Science \& Technology, Universiti Kebangsaan Malaysia, \\ Bangi 43600 UKM, Selangor, Malaysia \\ * Correspondence: ishak_h@ukm.edu.my
}

Received: 25 July 2020; Accepted: 25 August 2020; Published: 29 August 2020

\begin{abstract}
In this article, a study on the stability of Walter-B viscoelastic fluid in the highly permeable porous medium under the rotational speed modulation is presented. The impact of rotational modulation on heat transport is performed through a weakly nonlinear analysis. A perturbation procedure based on the small amplitude of the perturbing parameter is used to study the combined effect of rotation and permeability on the stability through a porous medium. Rayleigh-Bénard convection with the Coriolis expression has been examined to explain the impact of rotation on the convective flow. The graphical result of different parameters like modified Prandtl number, Darcy number, Rayleigh number, and Taylor number on heat transfer have discussed. Furthermore, it is found that the modified Prandtl number decelerates the heat transport which may be due to the combined effect of elastic parameter and Taylor number.
\end{abstract}

Keywords: convection; heat transfer; porous medium; weakly nonlinear analysis

\section{Introduction}

The natural convection through porous media has numerous applications in real-life problems. The utilization of porous media is in the oil reservoir, geophysics, and solidification of binary alloys. The comprehensive study on investigations on porous media and its applications are compiled very nicely in the excellent books of Ingham and Pop [1,2], Vafai [3,4], Straughan (2004), and Nield and Bejan [5]. Bég and Makinde [6] considered the upper convected Maxwell model on the viscoelastic fluid to examine the species transfer in high permeability porous media. They have reported that the Darcy number and Deborah number have a negligible effect on species diffusion.

Furthermore, many more articles are found based on the flow through porous media, some of them are: Malashetty and Basavaraja [7] studied the onset of convection in a horizontal anisotropic porous layer under thermal/gravity modulation. They have found that the temperature modulation gives rise to both sub-critical and super-critical motion, whereas gravity modulation always leads to the sub-critical motion. Shivakumara et al. [8] investigated an interesting practical problem based on the Darcy-Bénard-Marangoni convection with high permeability. They have considered the effective viscosity and fluid viscosity are different and found that the permeability constant stabilizing the onset convection. In addition, Shivakumara et al. [9], Postelnicu [10], Shivakumara and Sureshkumar [11], and Malashetty et al. [12] studied about the saturated porous medium.

Most of the engineering applications in fluid mechanics are restricted to a system in which externally imposed forces played an important role. These forces may be considered as temperature 
modulation, concentration modulation, rotational speed modulation, and others. The influence of external rotation is widely known due to significantly altering the nature of fluid flow by the Coriolis force. The investigation on convective heat transfer together with the rotational process in Newtonian fluids have practical importance. Research on rotational speed modulation is essential because this problem has been used in many branches of modern science such as enlarged production from petroleum reservoirs, geothermal energy utilization, atomic scraps repository rotating, insulation engineering, turbo-machinery, and bio-mechanics. The investigations on the effect of externally rotating force on thermal convection are initiated by Bénard [13] and Taylor [14-16] in the form of theoretical and experimental studies. The thermal and gravity modulations were initially generated by the rotational speed modulation from the original work of Donnelly [17]. Donnelly [17] analyzed the influence of rotational speed modulation on the thermal convection between two cylinders. Rauscher and Kelly [18] examined the effects of thermal and rotation modulation on the onset of convection.

In addition, the theoretical and experimental literature for rotational speed modulation was reported by Ahlers et al. [19], Niemela and Donnelly [20], and Walsh and Donnelly [21]. Bhadauria and Kiran [22] presented Rayleigh-Bénard convection of temperature-dependent viscosity of the fluid in the presence of internal heat source and rotational speed modulation. Gupta et al. [23] studied the effect of concentration/gravity modulation on mass transport in a rotating fluid layer. They have observed that the mass transport can govern the proper changes in amplitude and frequency of the modulation. Gupta [24] analyzed the effect of concentration modulation under the effect of applied magnetic field in a rotating fluid layer. Drury et al. [25] analyzed the impact of rotational speed modulation on Kepler AP star KIC2569073. Bowman et al. [26] observed the influence of rotational speed modulation to be chemically peculiar on stars by the photometry method. Recently, some articles have been published which use different types of modulation, for example, Keshri et al. [27-29], Kumar et al. [30], Jeong et al. [31], Tahir et al. [32], Saravanan and Kousalya [33], Roth et al. [34], Bhattacharya et al. [35], and Tayebi and Chamkha [36].

Since viscoelastic fluids are in demand nowadays due to their wide range of applications in modern technology and industries, such as petroleum engineering, reservoir engineering, material processing, biorheology, geophysics, and chemical engineering, so the studies on thermal instabilities on viscoelastic fluids are needed. Viscoelastic fluids have some silent features of instabilities like over-stability, which is not reflected in Newtonian fluids. Herbert [37] documented a report on the effect of elasticity on Couette flow. He performed a linear stability analysis and found that elasticity has a destabilizing effect on fluid flow. Furthermore, Vest [38] envisaged the natural convection in Maxwell fluid and obtained that the elasticity destabilizes the convection. He also reported that the convection could be slightly stabilized by considering rigid-rigid boundaries. Later on, Bhatnagar [39] used the approximation method to examine the stability in viscoelastic fluids. He observed that the neutral stability curves are more complicated in nature, as reported by Herbert [37] in the case of Couette flow. Several authors made reports on viscoelastic fluid-saturated porous media in the recent past. A few of them are: Postelnieu [10], Comissiong [40], Shivkumara et al. [41], Sheu [42], Rajib and Layek [43], Bhadauria and Kiran [44-46], Bhadauria and Kiran [47], and Bhadauria [48].

Landau [49] proposed an equation for evolution of magnitude of disturbance, which is reducible to a linear differential equation and is now called the Landau equation, in order to explain the transition to turbulence without providing valid proof. It is assumed that the foundation of nonlinear instability was laid by Landau (Drazin and Reid [50]). Later on, Stuart [51] has derived the equations claimed by Landau from hydrodynamic equations for Poiseuille flow. A detailed discussion on Ginzburg-Landau equations are documented in the Statistical Physics (Landau and Lifshitz [52]). They derived a Ginzburg-Landau equation to analyze the behavior of semiconductor in a magnetic field. They have used the general Landau theory of phase transition of the second kind which states that the difference between the symmetrical and unsymmetrical phase is described by the order of parameter, which will be zero at transition point. 
The above discussions show that investigation of rotational speed modulation on Walter-B viscoelastic fluids saturated in highly permeable porous media is required. Authors ensure that there is no document available that describes the impact of time-varying rotation on Walter-B viscoelastic fluids saturated in highly permeable porous media. With these motives, we have carried out a weakly nonlinear analysis of Walter-B viscoelastic fluids saturated in highly permeable porous media under a rotational speed modulation effect. A real Ginzburg-Landau equation has been obtained to depict the role of dimensionless parameters on heat transport.

\section{Mathematical Formulation}

In the present article, a Walter-B viscoelastic fluid enclosed within two parallel infinite flat plates saturated in highly permeable porous media is considered for the investigation. A Cartesian frame has been taken in such a way that the $x$-axis is along the lower plate and has an origin on it. The $z$-axis is taken perpendicular to the lower plate. For sustaining the fluid flow, an adverse temperature gradient is required; therefore, the upper plate is cooled and lower plate is heated. With a variable rotational speed $\Omega=(0,0, \Omega(t))$, the fluid layer is rotating about the $z$-axis (see Figure 1 ). The centrifugal force term has been neglected because the impact of rotation is limited to the Coriolis term. The Boussinesq approximation is employed which assumes that the density varies linearly with temperature. In view of the assumptions above, the basic governing model is given by:

$$
\begin{aligned}
& \nabla \cdot \vec{q}=0, \\
& \frac{\rho_{0}}{\phi}\left[\frac{\partial \vec{q}}{\partial t}+\frac{1}{\phi}(\vec{q} \cdot \nabla) \vec{q}+2(\vec{\Omega} \times \vec{q})\right]=-\nabla p+\rho \vec{g}-\frac{1}{K}\left(\mu-\mu_{v} \frac{\partial}{\partial t}\right) \vec{q}, \\
& \gamma \frac{\partial T}{\partial t}+(\vec{q} \cdot \nabla) T=k_{T} \nabla^{2} T, \\
& \rho=\rho_{0}\left[1-\alpha_{T}\left(T-T_{0}\right)\right] .
\end{aligned}
$$

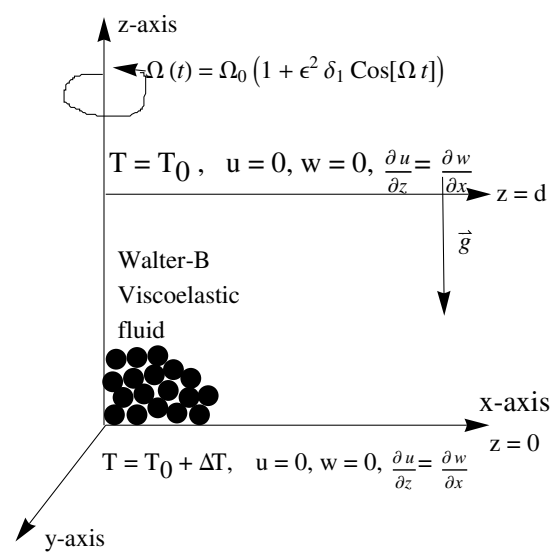

Figure 1. Schematic diagram of the problem geometry.

The examined temperature boundary situation at the plates is:

$$
T=T_{0}+\Delta T \quad \text { at } \quad z=0, \text { and } \quad T=T_{0} \quad \text { at } \quad z=d,
$$

where the variables have their usual meaning and are defined in the nomenclature section.

The present article focuses on the time-varying rotational speed modulation which alters the stability conditions and is defined as:

$$
\vec{\Omega}=\Omega_{0}\left[1+\epsilon^{2} \delta_{1} \cos (\Omega t)\right] \hat{k}
$$


where $\epsilon^{2} \delta_{1}$ is a very small quantity and is known as the amplitude of rotational speed modulation.

\section{Basic State}

The quantities at equilibrium position are defined as:

$$
\overrightarrow{q_{b}}=(0,0,0), \quad \rho=\rho_{b}(z), \quad p=p_{b}(z), \quad T=T_{b}(z) .
$$

Substituting Equation (7) into Equations (2)-(4), we get the following relation which helps us to define the basic state of pressure and temperature

$$
\begin{aligned}
& \frac{\partial p_{b}}{\partial z}=\rho_{b} \vec{g}, \\
& \frac{\partial^{2} T_{b}}{\partial z^{2}}=0,
\end{aligned}
$$

where subscript $b$ refers to the basic state. The solution of Equation (9) with Equation (5) is given by

$$
T_{b}=T_{0}+\Delta T\left(1-\frac{z}{d}\right) .
$$

To perform the stability analysis, an infinitesimal disturbance is created in the variables defined at the equilibrium position and then we will observe the effect of these small disturbances on the system. Let us define the variables at a perturbed state as

$$
\vec{q}=\vec{q}_{b}+\vec{q}^{\prime}, \quad \rho=\rho_{b}+\rho^{\prime}, \quad p=p_{b}+p^{\prime}, \quad T=T_{b}+T^{\prime},
$$

where ' shows the amount of the small deviation in the variables at basic state.

Substituting Equation (11) in Equations (2)-(4) and using basic state solution

$$
\begin{gathered}
\Delta \cdot \vec{q}^{\prime}=0, \\
\frac{\rho_{0}}{\phi}\left[\frac{\partial \overrightarrow{q^{\prime}}}{\partial t}+\frac{1}{\phi}\left(\overrightarrow{q^{\prime}} \cdot \nabla\right) \overrightarrow{q^{\prime}}+2\left(\vec{\Omega} \times \overrightarrow{q^{\prime}}\right)\right]=-\nabla p^{\prime}+\rho^{\prime} \vec{g}-\frac{1}{K}\left(\mu-\mu_{v} \frac{\partial}{\partial t}\right) \overrightarrow{q^{\prime}}, \\
\gamma \frac{\partial T^{\prime}}{\partial t}+\left(\overrightarrow{q^{\prime}} \cdot \nabla\right) T^{\prime}+w^{\prime} \frac{\partial T_{b}}{\partial z}=k_{T} \nabla^{2} T^{\prime}, \\
\rho^{\prime}=-\rho_{0} \alpha_{T} T^{\prime} .
\end{gathered}
$$

Now, we have taken the curl for eliminating the pressure term from Equation (13). In addition, we have considered all physical quantities to be independent of the $y$-coordinate as their axis. For two-dimensional convection, the stream function $\psi$ was introduced as $u^{\prime}=\frac{\partial \psi}{\partial z}, w^{\prime}=-\frac{\partial \psi}{\partial x}$. Furthermore, we write the resulting equations in dimensionless form by using the following transformation of variables:

$$
\left(x^{*}, y^{*}, z^{*}\right)=\frac{1}{d}(x, y, z), t=\frac{\phi d^{2}}{k_{T}} t^{*}, T^{\prime}=\Delta T T^{*}, \psi=k_{T} \psi^{*}, \Omega_{1}=\frac{k_{T}}{d^{2} \phi} \Omega_{2}^{*} .
$$

The dimensionless form of governing equations (after dropping the asterisks) is

$$
\begin{gathered}
\frac{1}{\operatorname{Pr}}\left[\frac{\partial\left(\nabla^{2} \psi\right)}{\partial t}-\frac{\partial\left(\psi, \nabla^{2} \psi\right)}{\partial(x, z)}\right]-\sqrt{\operatorname{Ta}} g_{m} \frac{\partial v}{\partial z}=-\frac{1}{D a}\left(1-\frac{\Gamma_{p}}{\operatorname{Pr}} \frac{\partial}{\partial t}\right) \nabla^{2} \psi-R a_{T} \frac{\partial T}{\partial x} \\
\frac{1}{\operatorname{Pr}}\left[\frac{\partial v}{\partial t}-\frac{\partial(\psi, v)}{\partial(x, z)}\right]+\sqrt{\operatorname{Ta}} g_{m} \frac{\partial \psi}{\partial z}=-\frac{1}{D a}\left(1-\frac{\Gamma_{p}}{\operatorname{Pr}} \frac{\partial}{\partial t}\right) v
\end{gathered}
$$




$$
M \frac{\partial T}{\partial t}-\frac{\partial(\psi, T)}{\partial(x, z)}-\frac{\partial \psi}{\partial x} \frac{d T_{b}}{d z}=\nabla^{2} T
$$

where $g_{m}=\left[1+\epsilon^{2} \delta_{1} \cos (\omega t)\right]$ and the dimensionless constants in the above equations are defined as: $\operatorname{Pr}=\frac{v \phi^{2}}{k_{T}}$, is the modified Prandtl number, $\sqrt{T a}=\frac{2 \Omega_{0} d^{2}}{v \phi}$, is the modified Taylor number, $D a=\frac{K}{d^{2}}$, is the Darcy number, $\Gamma_{p}=\frac{\mu_{v} \phi}{\rho_{0} d^{2}}$, is the elastic parameter, $R a_{T}=\frac{\alpha_{T} \Delta T g d^{3}}{v k_{T}}$, is the Rayleigh number, $M=\frac{\gamma}{\phi}$ is the modified thermal capacity ratio, and we have assumed $M=1$.

Similarly, the expression of $T_{b}(z)$ which arrives in Equation (18) can be collected through Equation (10) as

$$
\frac{d T_{b}}{d z}=-1
$$

Since stability analysis is performed for a short time, the time variation is rescaled as $\tau=\epsilon^{2} t$. Thus, Equations (16)-(18) can be obtained as

$$
\begin{aligned}
\frac{1}{D a} \nabla^{2} \psi+R a_{T} \frac{\partial T}{\partial x}-\sqrt{T a} \frac{\partial v}{\partial z}= & \frac{1}{\operatorname{Pr}} \frac{\partial\left(\psi, \nabla^{2} \psi\right)}{\partial(x, z)}-\frac{\epsilon^{2}}{\operatorname{Pr}}\left(1-\frac{\Gamma_{p}}{D a}\right) \frac{\partial \nabla^{2} \psi}{\partial \tau} \\
& +\epsilon^{2} \sqrt{T a} \delta_{1} \cos (\omega t) \frac{\partial v}{\partial z} \\
\frac{1}{D a} v+\sqrt{T a} \frac{\partial \psi}{\partial x}= & \frac{1}{\operatorname{Pr}} \frac{\partial(\psi, v)}{\partial(x, z)}-\frac{\epsilon^{2}}{\operatorname{Pr}}\left(1-\frac{\Gamma_{p}}{D a}\right) \frac{\partial v}{\partial \tau} \\
-\nabla^{2} T+\frac{\partial \psi}{\partial x}= & \frac{\partial(\psi, T)}{\partial(x, z)}-M \epsilon^{2} \frac{\partial T}{\partial \tau}
\end{aligned}
$$

The dimensionless thermal boundary conditions are given by

$$
\psi=\nabla^{2} \psi=T=0 \quad \text { at } \quad z=0,1 .
$$

From the Equations (20)-(22), one can recover the equations for uniform rotation reported by Vadasz [53] and Gupta et al. [54] for $\Gamma_{p}=0, \delta_{1}=0$.

\section{Finite Amplitude Equation and Heat Transfer}

To perform weakly nonlinear stability analysis, the asymptotic expansions method has been adopted. Thus, stream function $(\psi)$, rotational velocity $(v)$, temperature $(T)$, and Rayleigh number $\left(R a_{T}\right)$ are expanded in the power series of a small perturbation parameter $\epsilon \ll 1$. Accordingly, quantities are defined as

$$
\psi=\sum_{n=1}^{\infty} \epsilon^{n} \psi_{n}, v=\sum_{n=1}^{\infty} \epsilon^{n} v_{n}, T=\sum_{n=1}^{\infty} \epsilon^{n} T_{n}, R a_{T}=\sum_{n=1}^{\infty} \epsilon^{2 n-2} R a_{T_{2 n-2}},
$$

where $R a_{T_{0}}$ denotes the critical value of the Rayleigh number at which starting convection take place. Now, the above system of equation will be solved for different powers of $\epsilon$ by equating the similar terms of $\epsilon$ on both the sides, we get a series of partial differential equations at different orders of a perturbation parameter. 
4.1. First-Order System: Linear Stability Analysis

For the first-order problem, we have:

$$
\begin{aligned}
& \frac{1}{D a} \nabla^{2} \psi_{1}+R a_{T_{0}} \frac{\partial T_{1}}{\partial x}-\sqrt{T a} \frac{\partial v_{1}}{\partial z}=R_{11}, \\
& \frac{\partial}{\partial x} \psi_{1}-\nabla^{2} T_{1}=R_{12} \\
& \sqrt{T a} \frac{\partial \psi_{1}}{\partial z}+\frac{1}{D a} v_{1}=R_{13} .
\end{aligned}
$$

Here, $R_{11}, R_{12}$ and $R_{13}$ are independent of $\epsilon$ so $R_{11}=0, R_{12}=0$ and $R_{13}=0$. Thus, the solutions of Equations (25)-(27) satisfying the boundary conditions are obtained as:

$$
\left.\begin{array}{c}
\psi_{1}=A(\tau) \sin k x \sin \pi z \\
T_{1}=-\frac{\overline{\delta^{2}}}{A(\tau) \cos k x \sin \pi z} \\
v_{1}=-\sqrt{\operatorname{Tak} \pi D a A(\tau) \sin k x \cos \pi z}
\end{array}\right\},
$$

where $\delta^{2}=k^{2}+\pi^{2}$ and the value of amplitude $A(\tau)$ has to determine what is not possible here. It will be determined by applying the solvability condition at the third-order solution.

The expressions for the critical value of Rayleigh number and corresponding wave number are obtained as

$$
\begin{aligned}
R a_{T_{0}} & =\frac{1}{D a}\left[\frac{\delta^{4}+D a^{2} T a \pi^{2} \delta^{2}}{k^{2}}\right] \\
k_{0} & =\pi\left(1+T a D a^{2}\right)^{1 / 4} .
\end{aligned}
$$

When $T a=0$, the expression for critical Rayleigh number and wave number, which, given by Equations (29) and (30), reduces to

$$
\begin{aligned}
R a_{T_{0}} & =D a^{-1} \frac{\left(\pi^{2}+k^{2}\right)^{2}}{k^{2}} \\
k_{0} & =\pi
\end{aligned}
$$

The expressions in Equations (31) and (32) coincide with the classical result reported by Lapwood [55] for isotropic porous media.

\subsection{Second-Order System}

For the second-order problem, we have:

$$
\begin{aligned}
& \frac{1}{D a} \nabla^{2} \psi_{2}+R a_{T_{0}} \frac{\partial T_{2}}{\partial x}-\sqrt{T a} \frac{\partial v_{2}}{\partial z}=R_{21}, \\
& \frac{\partial}{\partial x} \psi_{2}-\nabla^{2} T_{2}=R_{22}, \\
& \sqrt{T a} \frac{\partial \psi_{2}}{\partial z}+\frac{1}{D a} v_{2}=R_{23},
\end{aligned}
$$

where $R_{21}, R_{22}$ and $R_{33}$ are obtained as follows:

$$
\left.\begin{array}{c}
R_{21}=0, \\
R_{22}=-\frac{A^{2} k^{2} \pi}{2 \delta^{2}} \sin 2 \pi z, \\
R_{23}=\frac{A^{2} k \sqrt{\frac{T a}{T a}} D a}{2 P r} \sin 2 k x,
\end{array}\right\}
$$


At this stage, the stream function, rotational velocity, and temperature profiles are independent of scaled time $\tau$. Thus, these profiles for the second-order system, subject to boundary conditions, can be obtained as:

$$
\left.\begin{array}{c}
\psi_{2}=0, \\
T_{2}=-\frac{k^{2}}{8 \pi \delta^{2}} A^{2}(\tau) \sin 2 \pi z, \\
v_{2}=\frac{\pi^{2} k \sqrt{T a D a^{2}}}{2 P r} A^{2}(\tau) \sin 2 k x,
\end{array}\right\}
$$

The local Nusselt number, $N u(\tau)$, for the stationary mode of convection is determined by:

$$
N u(\tau)=1+\frac{\left[\int_{0}^{\frac{2 \pi}{k}}\left(\frac{\partial T_{2}}{\partial z}\right) d x\right]_{z=0}}{\left[\int_{0}^{\frac{2 \pi}{k}}\left(\frac{\partial T_{b}}{\partial z}\right) d x\right]_{z=0}} .
$$

Now, putting the values of $T_{2}$ and $\frac{\partial T_{b}}{\partial z}$ into Equation (38), we get

$$
N u(\tau)=1+\frac{k^{2}}{4 \delta^{2}} A^{2}(\tau) .
$$

\subsection{Third-Order System}

Equate the coefficient of $\epsilon^{3}$ from Equations (20)-(22) to determine the solution at third order. After putting the expansions of $\psi, v$, and $T$, the following equations can be obtained:

$$
\begin{aligned}
& \frac{1}{D a} \nabla^{2} \psi_{3}+R a_{T_{0}} \frac{\partial T_{3}}{\partial x}-\sqrt{T a} \frac{\partial v_{3}}{\partial z}=R_{31} \\
& \frac{\partial}{\partial x} \psi_{3}-\nabla^{2} T_{3}=R_{32} \\
& \sqrt{T a} \frac{\partial \psi_{3}}{\partial z}+\frac{1}{D a} v_{3}=R_{33}
\end{aligned}
$$

where

$$
\begin{aligned}
R_{31}= & {\left[\frac{\delta^{2}}{P r}\left(1-\frac{\Gamma_{p}}{D a}\right) \frac{d A(\tau)}{d \tau}+\operatorname{Ta}^{2} A(\tau) D a \delta_{1} \cos (\omega t)\right.} \\
& \left.-\frac{R a_{T_{2}} k^{2} A(\tau)}{\delta^{2}}\right] \sin k x \sin \pi z, \\
R_{32}= & {\left[\frac{k}{\delta^{2}} \frac{d A(\tau)}{d \tau}-\frac{A^{3}(\tau) k^{3}}{4 \delta^{2}} \cos 2 \pi z\right] \cos k x \sin \pi z, } \\
R_{33}= & {\left[-\frac{A^{3}(\tau) \pi^{3} k^{2} \sqrt{T a} D a^{2}}{P r^{2}} \cos 2 k x+\frac{1}{P r}\left(1-\frac{\Gamma_{p}}{D a}\right) D a \sqrt{T a} \pi \frac{d A(\tau)}{d \tau}\right.} \\
& \left.-\delta_{1} \sqrt{T a} \cos (\omega t) A(\tau) \pi\right] \sin k x \cos \pi z .
\end{aligned}
$$

Concerning the occurrence of the solution of the third-order arrangement, a solvability condition has been imposed that results in terms of the time-periodic coefficients known as the real Landau equation for stationary convection in the form:

$$
A_{1} A^{\prime}(\tau)-A_{2} A(\tau)+A_{3} A^{3}(\tau)=0,
$$


where $A(\tau)$ is the unmodulated amplitude of heat convection. The coefficients $A_{1}, A_{2}, A_{3}$ are given by

$$
\begin{aligned}
& A_{1}=\left[\frac{T a \pi^{2} D a-\delta^{2}}{P r}\left(1-\frac{\Gamma_{p}}{D a}\right)-\frac{R a_{T_{0}} k^{2}}{\delta^{4}}\right] \\
& A_{2}=\left[2 \delta_{1} \operatorname{Ta}^{2} A(\tau) D a \cos (\omega t)-\frac{R a_{T_{2}} k^{2}}{\delta^{2}}\right] \\
& A_{3}=\left[\frac{T a \pi^{4} k^{2} D a^{3}}{2 P r^{2}}-\frac{R a_{T_{0}} k^{4}}{8 \delta^{4}}\right] .
\end{aligned}
$$

Equation (46) is reducible to the linear differential equation with the time-dependent coefficients; thus, determining its analytical solution is a difficult task. Therefore, a numerical solution has been obtained by using the in-built function NDSolve of Mathematica 11, subject to a suitable initial condition $A(0)=a_{0}$. In our calculations, assume that $R a_{T_{2}}=R a_{T_{0}}$ to keep the parameters to the minimum.

\section{Results and Discussion}

In this section, we have discussed the graphical representation of numerical outcomes in terms of heat transport versus slow time under rotational speed modulation of Walter-B viscoelastic fluid in highly permeable porous media. The study is performed under the weakly nonlinear stability theory, which indicates that the stability is possible by a certain choice of physical parameters. As we have known that many real-life applications complying with thermal convection under external regulatory forces are used for enhancing or inhibiting the heat transport in engineering and science, industry, food process, turbo-machinery, and chemical industry. Consequently, the rotational speed modulation as an external regulatory mechanism has been considered for the study of Walter-B viscoelastic fluid. The non-dimensional parameters that arise in the problem are $R a_{T}, \operatorname{Pr}, D a, T a, \delta_{1}, \omega$, and $\Gamma_{p}$. These parameters influence the convective heat transfer. For the range of the considered parameters, we have followed the articles of Shivkumara et al. [41], Beg and Makinde [6], and Kumar et al. [30]. Furthermore, as the Darcy number increases, the flow profile decreases due to the Darcian force decrease (Beg and Makinde [6]). Thus, a higher Darcy number has been considered to accelerate the flow formation. The common values of the parameters are taken as $\operatorname{Pr}=25, D a=0.3, \omega=2$, $\delta_{1}=0.05, \Gamma_{p}=0.5$, and $T a=20$ (unless otherwise stated). In all figures, the Nusselt number $(N u(\tau))$ concerning slow time $(\tau)$ under rotational speed modulation has been plotted for Walter-B viscoelastic fluid from Figure $2 \mathrm{a}-\mathrm{f}$.

In Figure 2a, the effect of Prandtl number on Nusselt number over slow time $(\tau)$ is depicted. As Pr enlarges, the Nusselt number decreases by considering other parameters at their common values. This is due to the momentum diffusivity dominating and, consequently, the temperature gradient decreasing. In addition, at a low value of $\mathrm{Pr}$, the heat transfer rate is higher and, at higher values of $\mathrm{Pr}$, the heat transfer is slow. Thus, Prandtl number stabilizes the system. This character opposes the result produced Bhadauria and Kiran [44] under gravity modulation. This is due to the combined effect of elastic parameter and rotational speed modulation. Figure $2 b$ illustrates the effect of frequency of Modulation $(\omega)$ on heat transport. Figure $2 b$ shows that, for a small value of $\omega$, the heat transfer is higher, while, at a large value of $\omega$, the heat transfer is less. As $\omega$ increases, the amplitude of modulation increases and consequently decreases the value of heat transfer. Therefore, the frequency of modulation $(\omega)$ stabilizes the system. The effect of modified Taylor number $(\mathrm{Ta})$ on the rate of heat transfer is depicted by Figure 2c. As Ta increases, $N u(\tau)$ increases by considering the other parameter at their common value. When rotation increases, then temperature gradient increases and therefore heat transfer increases. Figure $2 \mathrm{~d}$ scrutinizes the impact of amplitude of modulation $\left(\delta_{1}\right)$ on Nusselt number $(N u(\tau))$ for the common value of other parameters. The heat transport increases by increasing the value of $\delta_{1}$. Thus, we can find that $\delta_{1}$ is an external controlling mechanism of convection. Hence, the system becomes destabilized as $\delta_{1}$ increases. Figure 2e shows the variation of $N u(\tau)$ for different values of Darcy number $(D a)$. When $D a$ increases, the fluid particle moves easier in the progressively less fibrous porous region because the Darcian force resists the fluid flow and is inversely 
proportional to the Darcy number. Actually, this indicates that the Darcian force will decline for a larger permeability (higher $D a$ values). Thus, as permeability increases ( $D a$ increases), the heat transport increases so the Darcy number $(D a)$ has a destabilizing effect on the system as the rate of heat transfer increases. Figure $2 \mathrm{f}$ displays the variation of $N u(\tau)$ versus $\tau$ for different values of elastic parameter $\left(\Gamma_{p}\right)$. We found that an increase in the value of $\Gamma_{p}$ increases the value of Nusselt number $(N u(\tau))$ for a very short time. Thus, heat transport increases. At the same time, we also observe that the effect of the elastic parameter on the rate of heat transport diminishes as time passes.

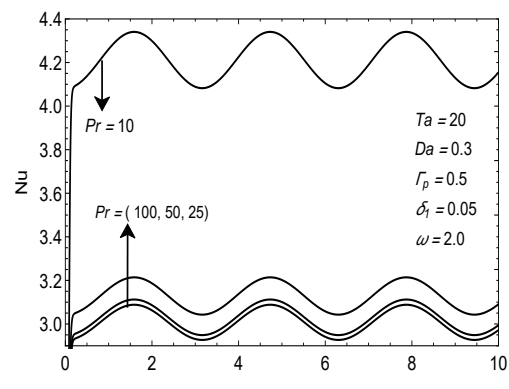

(a)

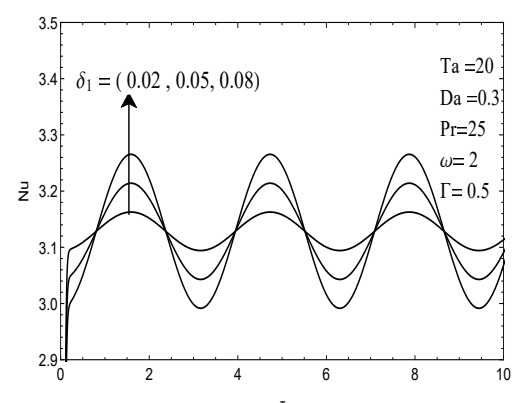

(d)

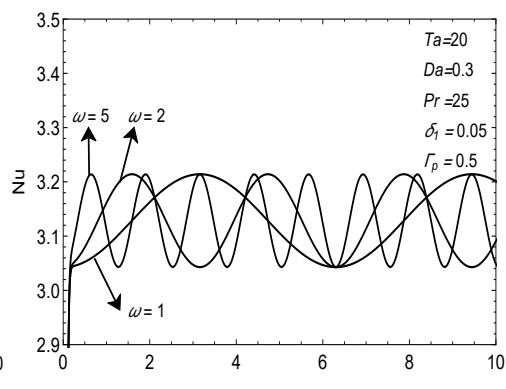

(b)

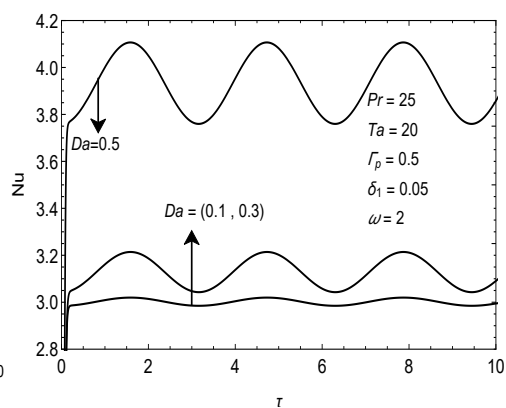

(e)

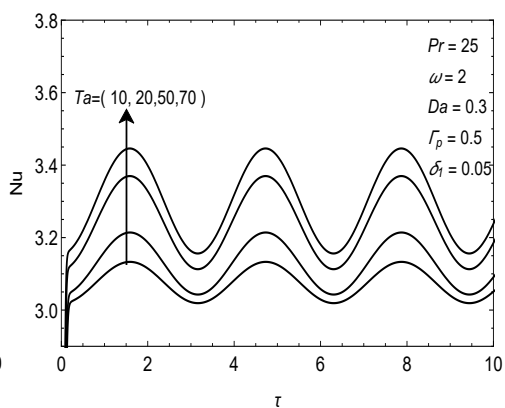

(c)

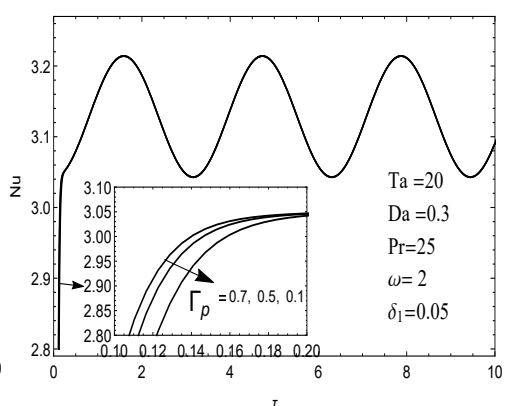

(f)

Figure 2. Variation of Nusselt number $(N u)$ with slow time $(\tau)$ for different values of parameters (a) $P r$, (b) $\omega$, (c) $T a,(\mathbf{d}) \delta_{1},(\mathbf{e}) D a,(\mathbf{f}) \Gamma_{p}$.

Figures 3 and 4 are plotted for the streamlines and corresponding isotherms respectively at different values of $\tau=0.1,1.0,2.0$ and 2.5 for the fixed value of other parameters. The figures show that, initially, heat transport is in the conduction state because of the small magnitude of streamlines and flat nature of isotherms. As the value of $\tau$ increases, we observe that the magnitude of streamlines increases and isotherms have more contour which confirms that the heat transfer is due to convection. Furthermore, we examine that the convection takes place more rapidly as time $(\tau)$ increases. From Figure $2 d$, we observe that contours are shifted towards the wall, which indicates that the system is approaching steady state. Figure $3 \mathrm{~d}$ shows that the contours become flat at $\tau=2.5$, which validates the results predicated by Figure $2 \mathrm{~d}$. 


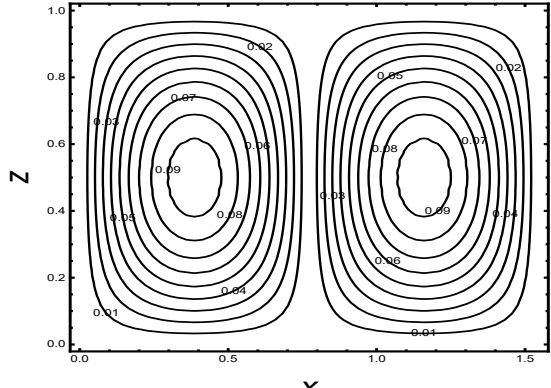

(a)

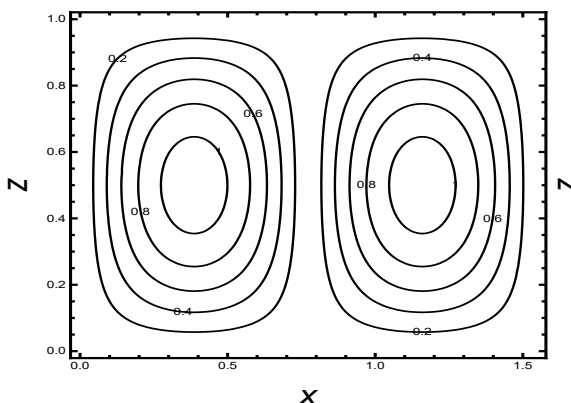

(c)

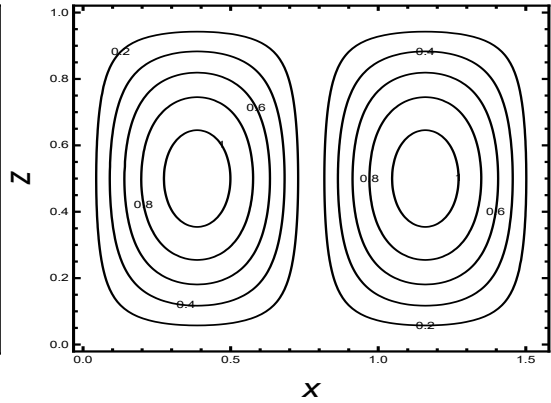

(b)

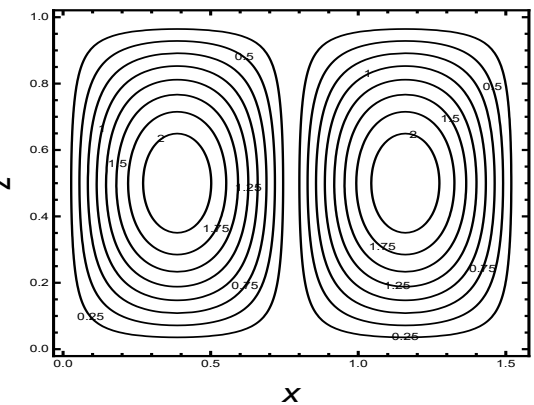

(d)

Figure 3. Streamlines for the fixed values of parameters at different scaled time (a) $\tau=0.1,(\mathbf{b}) \tau=1.0$, (c) $\tau=2.0$, (d) $\tau=2.5$ for the fixed values of $\operatorname{Pr}=25, D a=0.3, \omega=2, \delta_{1}=0.05, \Gamma_{p}=0.5$ and $T a=20$.

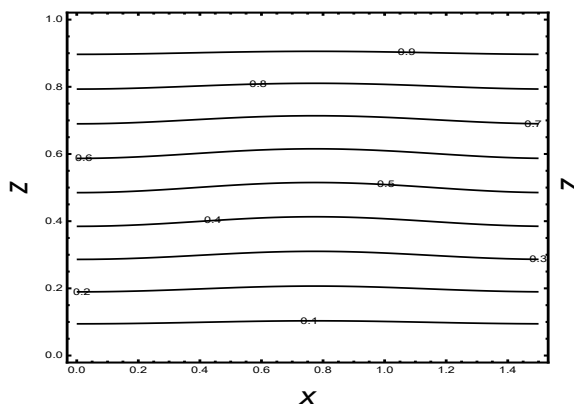

(a)

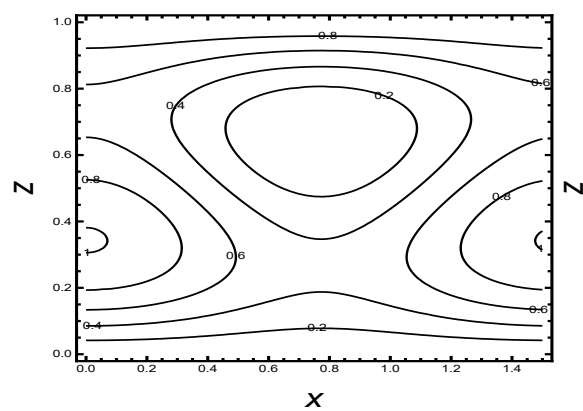

(c)

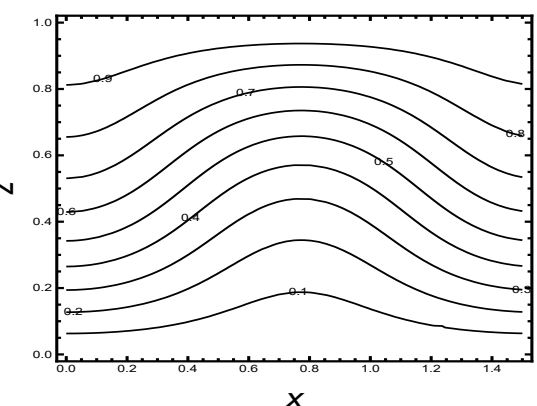

(b)

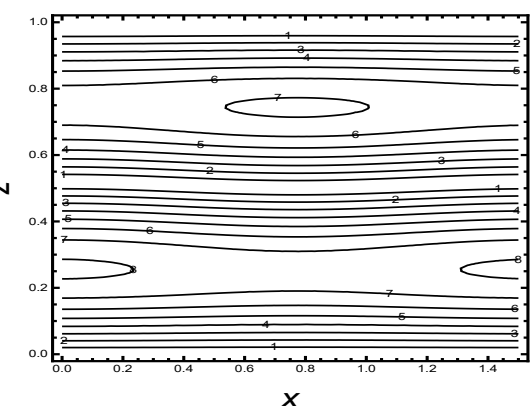

(d)

Figure 4. Isotherms for the fixed values of parameters at different scaled time (a) $\tau=0.1,(\mathbf{b}) \tau=1.0$, (c) $\tau=2.0$, (d) $\tau=2.5$ for the fixed values of $P r=25, D a=0.3, \omega=2, \delta_{1}=0.05, \Gamma_{p}=0.5$ and $T a=20$.

\section{Conclusions}

The present article displays the effect of modulated rotational speed in a highly permeable porous media saturated Walter-B viscoelastic fluid on Rayleigh-Bénard convection. In this study, an incompressible fluid saturated between two infinite parallel plates cooled from above and heated 
from below is considered. Here, a weekly nonlinear stability analysis has been presented by using a real Landau model in terms of the amplitude of convection. The impact of different dimensionless parameters is analyzed on the convective system, and we have drawn the following conclusions:

- The effect of the modified Prandtl number is to stabilize the convective system as $N u$ decreases with $\mathrm{Pr}$. This is the combined effect of elastic parameter and Taylor number.

- The effect of the increasing modified Taylor number $(T a)$ is to increase the heat transfer rate, and thus $\mathrm{Ta}$ destabilizes the system.

- The frequency of modulation $(\omega)$ stabilizes the system while the amplitude of modulation has the opposite effect.

- The heat transport can be accelerated with an increase in the Darcy number (i.e., rise in permeability) and hence the Darcy number destabilizes the convective system.

- Nusselt number increases with an increase in $\Gamma_{p}$ for an initial short period. After that, its effect is negligible on heat transport.

- At initially $(\tau=0.1)$, the heat transport is in a conduction mode and, when time $(\tau)$ increases, the conduction mode is switched into convection mode. Thus, at moderate values of time $(\tau)$, heat transport is carried due to convection. After $\tau=2.5$, we observe that the system arrives at a steady state.

We can summarize the above results as:

$$
\begin{gathered}
N u_{P r=100}<N u_{P r=50}<N u_{P r=25}<N u_{P r=10}, \\
N u_{T a=10}<N u_{T a=20}<N u_{T a=50}<N u_{T a=70}, \\
N u_{\delta_{1}=0.02}<N u_{\delta_{1}=0.05}<N u_{\delta_{1}=0.08}, \\
N u_{D a=0.1}<N u_{D a=0.3}<N u_{D a=0.5}, \\
N u_{\Gamma_{p}=0.1}<N u_{\Gamma_{p}=0.5}<N u_{\Gamma_{p}=0.7} .
\end{gathered}
$$

Author Contributions: Conceptualization, A.K., V.K.G. and N.M.; methodology, A.K., V.K.G. and N.M.; software, A.K., V.K.G. and N.M.; validation, A.K., V.K.G., N.M. and I.H.; formal analysis, A.K., V.K.G., N.M. and I.H.; investigation, A.K., V.K.G. and N.M.; resources, A.K., V.K.G. and N.M.; data curation, X.X.; writing-original draft preparation, A.K., V.K.G. and N.M.; writing-review and editing, A.K., V.K.G., N.M. and I.H.; funding acquisition, I.H. All authors have read and agreed to the published version of the manuscript.

Funding: We are grateful for the financial support received from the Universiti Kebangsaan Malaysia under the research grant GP-2019-K006388.

Acknowledgments: The authors are grateful to the referees for their most useful comments that helped refine the paper to the present form.

Conflicts of Interest: Authors declare no conflict of interest.

\section{Nomenclature}

\section{Latin Symbols}

A Amplitude of convection

$\delta_{1} \quad$ Amplitude of modulation

$\vec{g} \quad$ Gravitational acceleration, $(0,0,-g)$

$k \quad$ Wave number

$\vec{q} \quad$ Velocity Vector

$d \quad$ Depth between plates

$\mathrm{Nu} \quad$ Nusselt Number

$p \quad$ Pressure

$R a_{T} \quad$ Thermal Rayleigh Number $R a_{T}=\frac{\alpha_{T} g \Delta T d^{3}}{v k_{T}}$ 


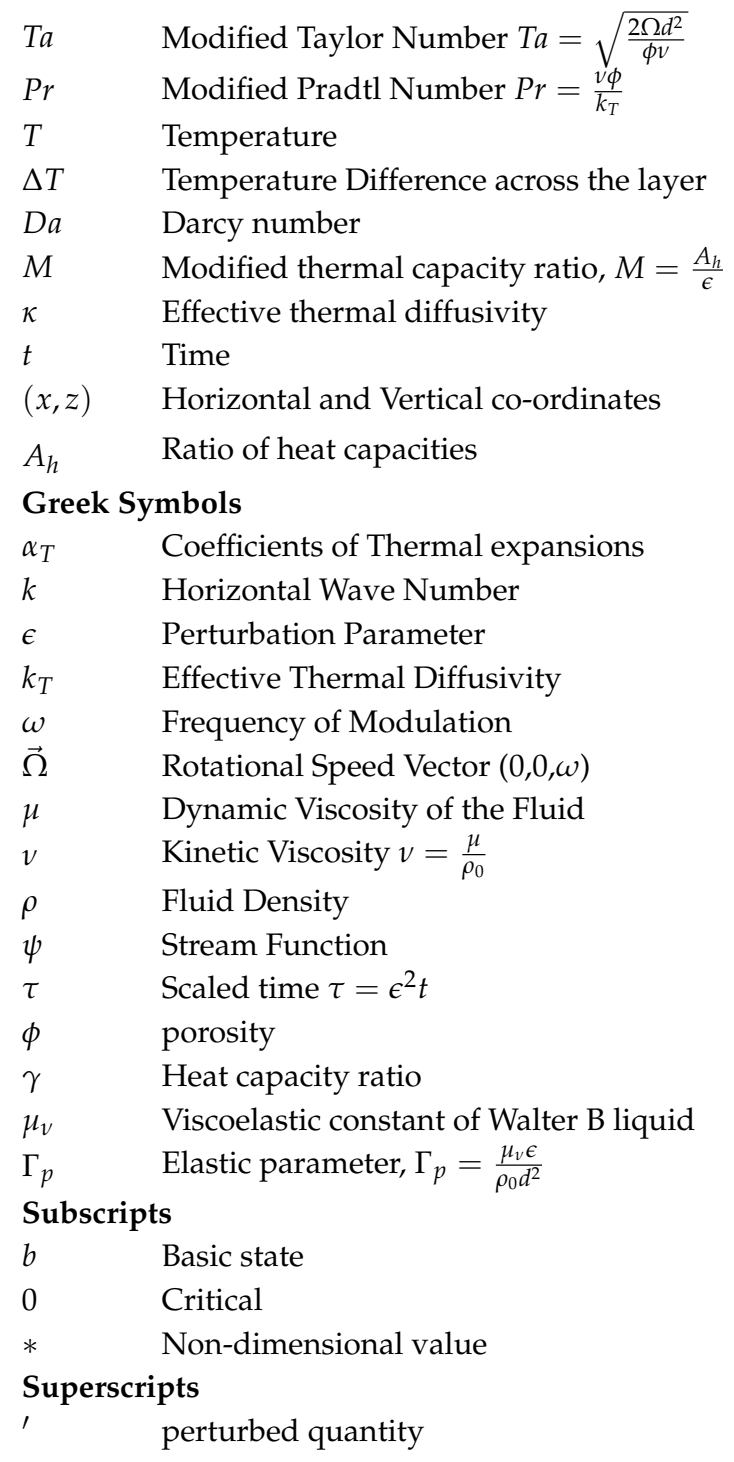

\section{References}

1. Ingham, D.B.; Pop, I. Transport Phenomena in Porous Media, 1st ed.; Pergamon: Oxford, UK, 1998.

2. Ingham, D.B.; Pop, I. Transport Phenomena in Porous Media, 3rd ed.; Elsevier: Oxford, UK, 2005.

3. Vafai, K. (Ed.) Handbook of Porous Media; Marcel Dekker: New York, NY, USA, 2000.

4. Vafai, K. (Ed.) Handbook of Porous Media, 2nd ed.; Taylor and Francis (CRC): Boca Raton, FL, USA, 2005.

5. Nield, D.A.; Bejan, A. Convection in Porous Media, 3rd ed.; Springer: New York, NY, USA, 2006.

6. Beg, O.A.; Makinde, O.D. Viscoelastic flow and species transfer in a Darcian high-permeability channel. J. Pet. Sci. Eng. 2011, 76, 93-99. [CrossRef]

7. Malashetty, M.S.; Basavaraja, D. The effect of thermal/gravity modulation on the onset of convection in a horizontal anisotropic porous layer. Int. J. Appl. Mech. Eng. 2003, 8, 425-439.

8. Shivakumara, I.S.; Nanjundappa, C.E.; Chavaraddi, K.B. Darcy-Benard-Marangoni convection in porous media. Int. J. Heat Mass Transf. 2009, 52, 2815-2823. [CrossRef]

9. Shivakumara, I.S.; Malashetty, M.S.; Chavaraddi, K.B. Onset of convection in a viscoelastic-fluid-saturated sparsely packed porous layer using a thermal nonequilibrium model. Can. J. Phys. 2006, 84, 973-990. [CrossRef]

10. Postelnicu, A. Thermal Hydrodynamic Instability of a Walters B Viscoelastic Fluid in a Fluid-Saturated Anisotropic Porous Medium with Fast Chemical Reaction; Eurotherm Seminar Reactive Heat Transfer in Porous Media: Ecole des Mines d'Albi, France, 2007; pp. 1-8. 
11. Shivakumara, I.S.; Sureshkumar, S. Convective instabilities in a viscoelastic-fluid-saturated porous medium with throughflow. J. Geophys. Eng. 2007, 4, 104-115. [CrossRef]

12. Malashetty, M.S.; Swamy, M.S.; Sidram, W. Thermal convection in a rotating viscoelastic fluid saturated porous layer. Int. J. Heat Mass Transf. 2010, 53, 5747-5756. [CrossRef]

13. Bénard, H. Les tourbillions cellulaires dans une nappe liquide transportant de la chaleur par convection en régime permanent. Annal. Chim. Phys. 1901, 23, 62-144.

14. Taylor, G.I. Motion of solids in fluids when the flow is not irrotational. Proc. R. Soc. Lond. A 1917, 93, 99-113.

15. Taylor, G.I. Experiments with rotating fluids. Proc. R. Soc. Lond. A 1921, 100, 114-121.

16. Taylor, G.I. The motion of a sphere in a rotating liquid. Proc. R. Soc. Lond. A 1922, 102, 180-189.

17. Donnelly, R.J. Experiments on the stability of viscous flow between rotating cylinders enhancement of stability by modulation. Proc. R. Soc. Lond. A 1964, 281, 130-139.

18. Rauscher, J.W.; Kelly, R.E. Effect of modulation on the onset of thermal convection in a rotating fluid. Int. J. Heat Mass Transf. 1975, 18, 1216-1217. [CrossRef]

19. Ahlers, G.; Hohenberg, P.C.; Lucke, M. Thermal convection under external modulation of the driving force. I. The Lorenz model. Phys. Rev. A 1985, 32, 3493-3518. [CrossRef] [PubMed]

20. Niemela, J.J.; Donnelly, R.J. Direct transition to turbulence in rotating Bénard convection. Phys. Rev. Lett. 1986, 57, 2524-2527. [CrossRef] [PubMed]

21. Walsh, T.J.; Donnelly, R.J. Taylor-Couette flow with periodically corotated and counterrotated cylinders. Phys. Rev. Let. 1988, 60, 700-703. [CrossRef] [PubMed]

22. Bhadauria, B.S.; Kiran, P. Effect of rotational speed modulation on heat transport in a fluid layer with temperature dependent viscosity and internal heat source. J. Ain Shams Eng. 2014, 5, 1287-1297. [CrossRef]

23. Gupta, V.K.; Kumar, A.; Singh, A.K. Analytical study of weakly nonlinear mass transfer in rotating fluid layer under time-periodic concentration/gravity modulation. Int. J. Non-Linear Mech. 2017, 97, 22-29. [CrossRef]

24. Gupta, V.K. Study of mass transport in rotating couple stress liquid under concentration modulation. Chin. J. Phys. 2018, 56, 911-921. [CrossRef]

25. Drury, J.A.; Murphy, S.J.; Derekas, A.; Sódor, A.; Stello, D.; Kuehn, C.A.; Bedding, T.R.; Bognár, Z.; Szigetr, L.; Szakáts, R.; et al. Large amplitude change in spot-induced rotational modulation of the kepler ap star kic2569073. Mon. Not. R. Astron. Soc. Sol. Stellar Astrophys. 2017, 471, 3193-3199. [CrossRef]

26. Bowman, D.M.; Buysschaert, B.; Neiner, C.; Pápics, P.I.; Oksala, M.E.; Aerts, C. K2 space photometry reveals rotational modulation and stellar pulsations in chemically peculiar a and b stars. Astron. Astrophys. 2018, 616,1-40. [CrossRef]

27. Keshri, O.P.; Kumar, A.; Gupta, V.K. Magento-solutal convection in Newtonian fluid layer with solutal modulated boundaries. Int. J. Non-Linear Mech. 2018, 107, 86-93. [CrossRef]

28. Keshri, O.P.; Kumar, A.; Gupta, V.K. Effect of internal heat Source on magnetostationary convection of couple stress fluid under magnetic field modulation. Chin. J. Phys. 2019, 57, 105-115. [CrossRef]

29. Keshri, O.P.; Gupta, V.K.; Kumar, A. Study of weakly nonlinear mass transport in Newtonian fluid with applied magnetic field under concentration/gravity modulation. Nonlinear Engg. 2019, 8, 513-522. [CrossRef]

30. Kumar, A.; Gupta, V.K. Study of heat and mass transport in couple stress liquid under G-gitter effect. Ain Shams Eng. J. 2018, 9, 973-984. [CrossRef]

31. Jeong, H.G.; Joon, K.B.; Sukyoon, L.; Bin, K.S.; Tackeun, K.; Hee-Joon, B.; Seung, B.J.; O-Ki, K.; Wan, O.C.; MOON-KU, H. Therapeutic temperature modulation against penumbral tissue loss in ischemic stroke. Crit. Care Med. 2020, 48, 353. [CrossRef]

32. Tahir, M.; Riaz, S.; Hussain, S.S.; Awan, A.; Xu, Y.B.; Naseem, S. Solvent mediated phase stability and temperature dependent magnetic modulation in $\mathrm{BiFeO}_{3}$ nanoparticles. J. Magn. Magn. Mater. 2020, 503, 166563. [CrossRef]

33. Saravanan, S.; Kousalya, M. On the onset of gravity modulated filtration convection in grade fluids via Mathieu functions. ASME J. Heat Transf. 2020, 142, 092701. [CrossRef]

34. Roth, S.; Zander, I.; Michelson, Y.; Ben-David, Y.; Banin, E.; Danielli, A. Identification of protein-protein interactions using a magnetic modulation biosensing system. Sens. Actuators B Chem. 2020, 303, 127228. [CrossRef]

35. Bhattacharyya, A.; Seth, G.S.; Kumar, R.; Chamkha, A.J. Simulation of Cattaneo-Christov heat flux on the flow of single and multi-walled carbon nanotubes between two stretchable coaxial rotating disks. J. Therm. Anal. Calorim. 2020, 139, 1655-1670. [CrossRef] 
36. Tayebi, T.; Chamkha, A.J. Entropy generation analysis during MHD natural convection flow of hybrid nanofluid in a square cavity containing a corrugated conducting block. Int. J. Numer. Methods Heat Fluid Flow 2019, 30, 1115-1136. [CrossRef]

37. Herbert, D.M. On the stability of viscoelastic liquids in heated plane Couette flow. J. Fluid Mech. 1963, 17, 353-359. [CrossRef]

38. Vest, C.M.; Arpaci, V.S. Overstability of a viscoelastic fluid layer heated from below. J. Fluid Mech. 1969, 36, 613-623. [CrossRef]

39. Bhatnagar, K.; Giesekus, H. On the stability of viscoelastic fluid flow-II. Rheol. Acta 1970, 9, 53-60. [CrossRef]

40. Comissiong, D.M.G.; Dass, T.D.; Ramkissoon, H.; Sankar, A.R. On thermal instabilities in a viscoelastic fluid subject to internal heat generation. Int. J. Math. Comput. Sci. 2011, 56, 826-833.

41. Shivakumara, S.; Lee, J.; Malashetty, M.S.; Sureshkumar, S. Effect of thermal modulation on the onset of convection in walters-B viscoelastic fluid-saturated porous medium. Trans. Porous Media 2011, 87, 291-307. [CrossRef]

42. Sheu, L.J. Linear stability of convection in a viscoelastic nanofluid layer, World Academy of Science. Eng. Technol. 2011, 58, 10-22.

43. Rajib, B.; Layek, G.C. The onset of thermo convection in a horizontal viscoelastic fluid layer heated underneath. Therm. Energy Power Eng. 2012, 1, 1-9.

44. Bhadauria, B.S.; Kiran, P. Weak nonlinear oscillatory convection in a viscoelastic fluid layer under gravity modulation. Int. J. Nonlinear Mech. 2014, 65, 133-140. [CrossRef]

45. Bhadauria, B.S.; Kiran, P. Weakly nonlinear oscillatory convection in a viscoelastic fluid saturating porous medium under temperature modulation. Int. J. Heat Mass Transf. 2014, 77, 843-851. [CrossRef]

46. Bhadauria, B.S.; Kiran, P. Weak nonlinear oscillatory convection in a viscoelastic fluid-saturated porous medium under gravity modulation. Trans. Porous Media 2014, 104, 451-467. [CrossRef]

47. Bhadauria, B.S.; Kiran, P. Chaotic and oscillatory magneto-convection in a binary viscoelastic fluid under G-jitter. Int. J. Heat Mass Transf. 2015, 84, 610-624. [CrossRef]

48. Bhadauria, B.S. Chaotic convection in a viscoelastic fluid saturated porous medium with a heat source. J. Appl. Math. 2016, 1487616. [CrossRef]

49. Landau, L.D. On the problem of turbulence. Dokl. Akad. Nauk SSSR 1944, 44, 339-349.

50. Drazin, P.G.; Reid, W.H. Hydrodynamic Stability, 2nd ed.; Cambridge University Press: Cambridge, UK, 2004.

51. Stuart, J.T. On the nonlinear mechanics of hydrodynamic stability. J. Fluid Mech. 1958, 4, 1-21. [CrossRef]

52. Landau, L.D.; Lifshitz, E.M. Statistical Physics-Part (2); Pergamon Press Ltd.: Oxford, UK, 1969.

53. Vadasz, P. Heat transfer and fluid flow in rotating porous media. Dev. Water Sci. 2002, 47, 469-476.

54. Gupta, V.K.; Bhadauria, B.S.; Hashim, I.; Jawdat, J.; Singh, A.K. Chaotic convection in rotating fluid layer. Alex. Eng. J. 2015, 54, 981-992. [CrossRef]

55. Lapwood, E.R. Convection of a fluid in a porous medium. Mathe. Proc. Camb. Philos. Soc. 1948, 44, 508-521. [CrossRef]

(C) 2020 by the authors. Licensee MDPI, Basel, Switzerland. This article is an open access article distributed under the terms and conditions of the Creative Commons Attribution (CC BY) license (http:// creativecommons.org/licenses/by/4.0/). 